\title{
A utilização de recursos educacionais digitais e o ensino do campo aditivo: uma
}

\section{revisão sistemática da literatura}

The use of digital educational resources and the teaching of the additive field: a systematic

\author{
literature review
}

El uso de recursos educativos digitales y la enseñanza del campo aditivo: una revisión sistemática de la literatura

Recebido: 03/10/2021 | Revisado: 10/10/2021 | Aceito: 13/10/2021 | Publicado: 16/10/2021

\author{
Nassara Maia Cabral Cardoso Gomes \\ ORCID: https://orcid.org/0000-0003-4675-2315 \\ Instituto Federal de Educação, Ciência e Tecnologia do Ceará, Brasil \\ E-mail: nassaramaia@gmail.com \\ Francisco Ellivelton Barbosa \\ ORCID: https://orcid.org/0000-0002-2918-4902 \\ Instituto Federal de Educação, Ciência e Tecnologia do Ceará, Brasil \\ E-mail: ellivelton.barbosa@hotmail.com \\ Juscileide Braga de Castro \\ ORCID: https://orcid.org/0000-0002-6530-4860 \\ Universidade Federal do Ceará, Brasil \\ E-mail: juscileide@ virtual.ufc.br
}

\begin{abstract}
Resumo
O presente artigo objetiva realizar uma Revisão Sistemática de Literatura (RSL) de teses e dissertações dos últimos 10 anos que apresentem como campo de pesquisa o uso de Recurso Educacional Digital (RED), bem como o ensino de conceitos voltados a adição e subtração, ou seja, as estruturas aditivas. Foi realizada uma RSL mista de convergência qualitativa. O Portal de busca das pesquisas foi o Portal de Teses e Dissertações da CAPES, analisando um total de 9 pesquisas, dentre as 27 encontradas, pois se enquadraram nas palavras utilizadas na busca. Diante dessa análise, constatou-se a necessidade do surgimento de novas pesquisas que apresentem o uso do RED no ensino do campo aditivo, tendo em vista a necessidade em ensinar conceitos relacionados ao campo aditivo utilizando o RED pode favorecer no ensino e aprendizagem. Espera-se que esta pesquisa contribua para a difusão de novas pesquisas que abordam o tema discutido.
\end{abstract}

Palavras-chave: Campo aditivo; Recurso educacional digital; Revisão sistemática de literatura.

\begin{abstract}
This article aims to carry out a Systematic Literature Review (RSL) of theses and dissertations from the last 10 years that present as a research field the use of Digital Educational Resource (RED), as well as the teaching of concepts aimed at addition and subtraction, or that is, the additive structures. A mixed RSL of qualitative convergence was performed. The search portal for the research was the CAPES Theses and Dissertations Portal, analyzing a total of 9 researches, among the 27 found, as they fit the words used in the search. Given this analysis, it was found that there is a need for the emergence of new research that present the use of RED in teaching the additive field, in view of the need to teach concepts related to the additive field using RED can favor teaching and learning. It is expected that this research will contribute to the dissemination of new research that address the topic discussed.
\end{abstract}

Keywords: Additive field; Digital educational resource; Systematic literature review.

\section{Resumen}

Este artículo tiene como objetivo realizar una Revisión Literaria Sistemática (RSL) de tesis y disertaciones de los últimos 10 años que presentan como campo de investigación el uso del Recurso Educativo Digital (RED), así como la enseñanza de conceptos orientados a la suma y resta. , es decir, las estructuras aditivas. Se realizó una RSL mixta de convergencia cualitativa. El portal de búsqueda de la investigación fue el Portal de Tesis y Disertaciones CAPES, analizando un total de 9 investigaciones, entre las 27 encontradas, según se ajustan a las palabras utilizadas en la búsqueda. Ante este análisis, se encontró que existe la necesidad de que surjan nuevas investigaciones que presenten el uso de RED en la enseñanza del campo aditivo, en vista de la necesidad de enseñar conceptos relacionados con el campo aditivo que el uso de RED pueda favorecer la enseñanza y el aprendizaje. . Se espera que esta investigación contribuya a la difusión de nuevas investigaciones que aborden el tema discutido.

Palabras clave: Campo aditivo; Recurso educativo digital; Revisión sistemática de la literatura. 


\section{Introdução}

Com uma frequência considerável, pesquisadores participam de um ambiente globalizado e dinâmico, onde é exigida a capacidade em tomar decisões com eficácia, apresentando reconhecimento e destaque nas suas publicações. Dentre as decisões apresentadas no processo desenvolvido em uma pesquisa, a escolha metodológica para selecionar os estudos mais relevantes ao tema pesquisado constitui fator decisivo no desenvolvimento da publicação, sendo ainda um grande desafio para pesquisadores ao redor do mundo (Caiado et al, 2016).

Realizar uma Revisão de Literatura é de considerável importância na elaboração de trabalhos acadêmicos e científicos. Revisar a literatura evita o surgimento de duplicação de pesquisas, bem como o reaproveitamento e a aplicação de pesquisas em contextos e escalas distintas. É possível também observar falhas nos estudos, conhecer recursos importantes na construção de um estudo com características específicas, desenvolver estudos que cubram falhas na literatura apresentando contribuição eficaz em um determinado campo; propor problemas, temas, metodologias e hipóteses de pesquisa; facilitar os recursos disponíveis a favor da sociedade, do meio científico, das instituições e governos que financiam a Ciência (Galvão, \& Ricarte, 2019).

Destarte, a Revisão Sistemática de Literatura (RSL) é caracterizada por uma questão explicita, claramente definida como uma pesquisa abrangente e sistemática de estudos, uma extração de dados, análise apropriada, apresentação dos resultados, interpretações ancoradas por dados, bem como implicações para futuras pesquisas (Ravindran, \& Shankar, 2015).

A RSL é uma modalidade de pesquisa que está focada no trabalho de reprodutibilidade de outros pesquisadores contendo, de modo evidente, as bases de dados bibliográficas que foram consultadas, as estratégias de busca escolhidas, o processo de seleção das pesquisas, os critérios de inclusão e de exclusão destas pesquisas, bem como o processo de análise dos mesmos (Galvão, \& Ricarte, 2019).

O presente artigo objetiva realizar uma revisão sistemática de literatura de dissertações e teses brasileiras dos últimos 10 anos que apresenta como campo de pesquisa o uso de Recursos Educacionais Digitais (RED) e o ensino do Campo Aditivo. Pretende-se, assim, descobrir pesquisas que evidenciem o uso do RED no ensino de Matemática, sobretudo o Campo Aditivo.

Entendemos a importância do conhecimento matemático, pois sua aplicação nas atividades cotidianas não se limita apenas à contagem, medição de objetos, grandezas, bem como técnicas de cálculo com os números e grandezas. Estuda-se, também, a incerteza de fenômenos aleatórios (Brasil, 2017).

A Matemática faz parte do currículo escolar e a sua compreensão é um desafio para estudantes e professores. Pesquisas identificaram as causas dessas dificuldades, traçando possíveis soluções para desmistificar conceitos equivocados sobre a Matemática (Souza, 2015; Castro, 2016).

O último resultado do Sistema de Avaliação da Educação Básica (SAEB) aponta que o conhecimento da Matemática deve ser apresentado por meio da resolução de problemas, considerando capacidades como estabelecimento de relações, observação, comunicação em diferentes linguagens, validação de processos e argumentação, objetivando as diferentes maneiras de raciocínio, tais como: intuição, dedução, indução e estimativa (Brasil, 2019).

Apesar destas indicações, os resultados mostram que 51,1\% dos estudantes do $5^{\circ}$ ano do Ensino Fundamental não são capazes de "Determinar o resultado da subtração, com recursos à ordem superior, entre números naturais de até cinco ordens, utilizando as ideias de retirar e comparar"; assim como 69,4\% destes mesmos estudantes não conseguem: "Resolver problemas que envolvam soma e subtração de valores monetários; Resolver problemas que envolvam a composição e a decomposição polinomial de números naturais de até cinco ordens" (Brasil, 2019, p. 54-58, p. 98). Estes resultados indicam que muitos estudantes finalizam o $5^{\circ}$ ano com dificuldades em conceitos do campo aditivo.

Além das dificuldades apresentadas no que tange ao ensino de Matemática, em especial ao Campo Aditivo, pesquisas (Castro, 2016; Castro Filho et al, 2008) indicam que as Tecnologias Digitais de Comunicação e Informação (TDIC) podem 
contribuir com a aprendizagem matemática. Dentre as TDIC pode-se mencionar o uso dos Recursos Educacionais Digitais (RED), explorando, assim, diferentes maneiras de se ensinar e aprender, em especial, os conceitos relacionados ao campo aditivo.

A seguir, será apresentado o referencial teórico da pesquisa, seguido do percurso metodológico, em que serão apresentadas a RSL das teses e dissertações encontradas, bem como o levantamento e discussão das pesquisas. Por fim, têm-se as conclusões e referências deste artigo.

\section{O Campo Aditivo e os Recursos Educacionais Digitais}

De acordo com (Vergnaud, 2009), uma única situação possui um conjunto de conceitos, assim como também não é possível isolar um conceito, o que justifica a determinação de campos conceituais. Um campo conceitual é formado por um conjunto de situações, representações, procedimentos, propriedades, relações, dentre outros elementos.

O Campo Aditivo, por exemplo, envolve situações com a ideia de juntar, separar, acrescentar, retirar, completar e comparar; portanto, pode envolver adições ou subtrações. Há ainda que se considerar que o campo aditivo pode ter interseções com outros campos, como o multiplicativo e o algébrico, o que pode tornar as situações mais complexas (Castro, 2016).

Por isso, (Vergnaud, 1996) explica que para a aprendizagem das situações do campo aditivo é necessário explorar um conjunto de situações em que o tratamento requer uma ou várias adições, subtrações, inclusive, a combinação das duas operações.

Caso essa aprendizagem não ocorra, existirá dificuldade em construir o conhecimento matemático posterior, ou seja, entender conceitos matemáticos mais complexos. Em vista disso, é importante que os professores compreendam o porquê o aluno apresenta dificuldades na aprendizagem dessas situações (Magina et al, 2010).

Além de compreender as dificuldades, é preciso explorar recursos didáticos e metodologias que proporcionem o desenvolvimento conceitual. Dentre as possibilidades têm-se as tecnologias que podem proporcionar novas oportunidades de comunicação e interação a partir do uso e da criação de imagens, de sons, de animações e da combinação de tais materiais (Rojo, \& Moura, 2012; Lira, Leitão, \& Castro, 2019).

$\mathrm{O}$ advento da Educação entreposta por computadores e a consequente utilização de Recursos Educacionais Digitais, pode proporcionar um cenário importante para novas pesquisas, pois interfere no funcionamento e desenvolvimento qualitativo dos ambientes de ensino e de aprendizagem. Cresce, no Brasil, o investimento de educadores e instituições de nível superior em pesquisa, bem como a produção de RED (Afonso et al., 2011).

O uso de tecnologias com o foco na Educação apresenta vantagens, quais sejam: produção de conteúdos (Lira, Leitão, \& Castro, 2019), representação de gráficos, simulação de situações reais, facilidade de representação e visualização de gráficos, múltiplas representações, dentre outros (Castro, 2012, 2016). Dependendo da mediação, as tecnologias podem oportunizar a construção de significados, possibilitando o engajamento dos estudantes (Castro, 2012).

Quando bem utilizados, os RED podem ser aliados ao processo educativo. Para que isso ocorra, é necessário que o professor identifique quais objetivos precisam ser alcançados e, com isso, definir as estratégias para utilizar o recurso na aula (Braga, \& Menezes, 2014). A facilidade de reprodução, manipulação e acesso aos RED tem favorecido o crescimento das redes de aprendizado formais, a exemplo de escolas e universidades (Amiel, \& Soares, 2015).

Ressalta-se ainda que a Base Nacional Comum Curricular (BNCC), documento normativo para a construção dos currículos das escolas brasileiras, destacam habilidades e competências que requisitam o uso de Tecnologias Digitais (Brasil, 2017). Na atualidade, devido a pandemia causada pelo novo Coronavírus, as Tecnologias Digitais, ou seja, os RED têm sido cada vez mais requisitados como recurso para o ensino e a aprendizagem (Hodges et al, 2020).

De acordo com as discussões realizadas, apresenta-se, a seguir, os procedimentos metodológicos para esta RSL. 


\section{Percurso da RSL e apresentação das pesquisas}

A análise realizada se deu por uma RSL mista de convergência qualitativa. Segundo (Galvão \& Ricarte, 2019), este tipo de revisão transforma os resultados de estudos qualitativos, quantitativos e de estudos adotando métodos mistos em resultados qualitativos. Tal revisão pode ser aplicada em estudos que possuem amostras pequenas e estão direcionadas para desenvolver, refinar e revisar um dado quadro conceitual.

A RSL foi realizada em repositório de busca das teses e dissertações, ou seja, no Catálogo de Teses \& Dissertações da Capes. Para uma melhor organização, utilizou na busca as palavras entre aspas e separadas por vírgula, quais sejam: “Recurso Educacional Digital" e "Campo Aditivo". Foram selecionados, também, o ano (2011 a 2020), tendo em vista que a temática relacionada aos RED é recente, carecendo, assim, de pesquisas atuais. Dentre o campo "áreas do conhecimento", foram removidas as áreas de Geografia e Sociais e Humanidades.

O total de pesquisas encontradas, considerando os filtros aplicados, foram 27. Os resumos dessas pesquisas foram lidos, objetivando saber se contemplavam os temas de interesse, ou seja, RED e Campo Aditivo. Entretanto, 18 pesquisas não foram analisadas, pois 8 desse total não possuíam divulgação autorizada na plataforma Sucupira; 1 pesquisa estava relacionada ao uso das tecnologias na Arte/Educação, fugindo, assim, dos objetivos deste artigo; 8 trabalhos são anteriores à plataforma Sucupira e, por isso, não estavam disponíveis: 1 abordava apenas o campo multiplicativo.

Desta forma, foram analisadas 9 pesquisas, dentre 6 dissertações e 3 teses. Tais pesquisas foram organizadas no Quadro 1, contendo: Código (D para dissertação e T para tese), ano, autor(a) e título. Será apresentado um recorte das pesquisas encontradas, por tópicos, quais sejam: objetivos, foco (sujeitos), referencial teórico, tipo de pesquisa/metodologia e os resultados.

Quadro 1. Levantamento de dissertações e teses - 2011 a 2020.

\begin{tabular}{|c|c|c|c|}
\hline Código & Ano & Autor & Título \\
\hline D1 & 2015 & Daniela Batista Santos & $\begin{array}{l}\text { Ensino de Matemática crítico e reflexivo: a Teoria das Situações Didáticas como estratégia } \\
\text { para a aprendizagem das operações básicas da aritmética na rede municipal de ensino. }\end{array}$ \\
\hline D2 & 2014 & $\begin{array}{l}\text { Danielly Regina Kaspary } \\
\text { dos Anjos }\end{array}$ & $\begin{array}{l}\text { Uma análise praxeológica das operações de adição e subtração de números naturais em uma } \\
\text { coleção de livros didáticos dos anos iniciais do ensino fundamental. }\end{array}$ \\
\hline D3 & 2013 & $\begin{array}{l}\text { José Fernando Fernandes } \\
\text { Pereira }\end{array}$ & $\begin{array}{c}\text { Resolução de problemas do campo aditivo por alunos de quinto ano de uma escola pública da } \\
\text { cidade de São Paulo. }\end{array}$ \\
\hline D4 & 2013 & Caroline Adjane Fiore & $\begin{array}{l}\text { Os pensamentos narrativo e lógico-científico na resolução de problemas nos campos } \\
\text { conceituais aditivo e multiplicativo no ano final do ensino fundamental I }\end{array}$ \\
\hline D5 & 2014 & Gabriele Bonotto Silva & Teoria dos Campos Conceituais, habilidades e competências: uma experiência de ensino \\
\hline D6 & 2017 & $\begin{array}{l}\text { Raphael de Oliveira } \\
\text { Freitas }\end{array}$ & Tecnologias móveis na formação de professores que ensinam Matemática. \\
\hline $\mathrm{T} 1$ & 2015 & $\begin{array}{l}\text { Maria Emília Melo } \\
\text { Tamanini Zanquetta }\end{array}$ & Uma investigação com alunos surdos do ensino fundamental: o cálculo mental em questão \\
\hline $\mathrm{T} 2$ & 2014 & Rosineide de Sousa Jucá & $\begin{array}{l}\text { Um estudo das competências e habilidades na resolução de problemas aritméticos aditivos e } \\
\text { multiplicativos com os números decimais }\end{array}$ \\
\hline $\mathrm{T} 3$ & 2017 & Sandra Cristina Lopes & $\begin{array}{c}\text { Comunidade de prática: resolução de problemas profissionais sobre o ensino de relações } \\
\text { contextuais }\end{array}$ \\
\hline
\end{tabular}

Fonte: Autores (2021). 
A pesquisa D1, realizada por (Santos, 2015), objetivou desenvolver conceitos das operações básicas de maneira crítica e reflexiva à luz da Teoria das Situações Didáticas (TSD). Teve como sujeitos professores da Rede Municipal de Alagoinhas/Ba. Os principais referenciais teóricos adotados na pesquisa são: Brousseau, Vergnaud, Santana, Magina et al, Lara, Artigue.

Como metodologia, adotou a pesquisa qualitativa de intervenção do tipo participante, baseados nos princípios da Engenharia Didática e produziu os dados da sua pesquisa através da: aplicação de um questionário-sondagem; Análise das atividades desenvolvidas em um curso de formação; Questionário reflexivo sobre as operações; Entrevistas semiestruturadas. Em sua pesquisa, apresentou as tecnologias, principalmente a utilização do software educacional, que é um recurso importante no ensino e aprendizagem de Matemática. Os resultados desta pesquisa apontaram que a TSD propiciou a percepção da importância em questionar, permitindo ao aluno a construção do seu conhecimento sob a perspectiva da autonomia voltada ao ensino de Matemática. Foi percebido a existência de avanços conceituais no campo aditivo, bem como na prática pedagógica, com destaque nas ações utilizadas no desenvolvimento das atividades propostas no curso e na sala de aula.

D2 apresentou como objetivo caracterizar o ensino das operações de adição e de subtração em uma coleção dos livros didáticos dos cinco primeiros anos escolares (Anjos, 2015). O principal referencial teórico desta pesquisa foi a Teoria dos Campos Conceituais, especificamente, as estruturas aditivas. A metodologia de análise do livro didático se deu a partir da Teoria Antropológica do Didático, envolvendo aspectos matemáticos e didáticos do ensino investigativo. Por se tratar de análise do livro didático, os elementos tecnológicos não foram alvos de estudo, embora tenha adotado a praxeologia matemática de (Chevallard, 1998). Por fim, os resultados evidenciaram a valorização pela prática e ensino de diferentes técnicas de resolução, a busca da institucionalização dos algoritmos usuais das operações de adição e subtração e o descaso de determinados ostensivos em detrimento de outros que se apresentam mais abrangentes, a fim de responder às tarefas apresentadas e mais enxutas no que concerne o caráter instrumental na atividade matemática.

D3 objetivou indicar saberes e dificuldades apresentadas por discentes do 5o ano, na resolução de situações do campo aditivo. A fundamentação teórica se baseou na Teoria dos Campos Conceituais de Gerárd Vergnaud e colaboradores estrangeiros e brasileiros. As metodologias adotadas foram a pesquisa qualitativa e quantitativa, estruturadas no confronto das dificuldades dos estudantes, o referencial teórico, as questões de pesquisa e os apontamentos propostos no referencial teórico. Os resultados de (Pereira, 2013) foi a possibilidade em apresentar indicadores de que os alunos lêem e compreendem os enunciados das situações-problema, mas erram os algoritmos. Apresenta que a percepção das dificuldades dos alunos em resolver as situações do campo aditivo estão ligadas a falta de congruência semântica entre o enunciado e a operação para se resolver a situação. Apresentou também, como resultado prático, houve o retorno do pesquisador à escola onde aconteceu a pesquisa, apresentando os resultados desta aos sujeitos, refletindo as práticas escolhidas e sugerindo alternativas com base no referencial teórico utilizado.

D4 apresentou como objetivos observar e analisar as estratégias na resolução de problemas dos campos aditivo e multiplicativo por estudantes do $5^{\circ}$ ano do Ensino Fundamental de uma escola Estadual da cidade de São Paulo. A teoria principal adotada na pesquisa foi a Teoria dos Campos Conceituais na escolha e classificação das situações-problema. Já a análise dos modos de pensamento narrativo e lógico-científico foram baseadas no trabalho de Bruner. Como metodologia, foi escolhido um estudo do tipo levantamento ou survey, proposto por (Fiorentini \& Lorenzato, 2006). Como resultados, (Fiore, 2013) percebeu e observou que os estudantes obtiveram um desenvolvimento satisfatório nas resoluções apresentadas,bem como na diversidade de níveis de classificação das situações-problema propostas. Foi percebido também que o professor, ao conduzir a orientação na resolução de problemas, poderá auxiliar o aluno a organizar o pensamento lógico no que diz respeito à interpretação dos dados, conceitos matemáticos, processo do algoritmo e a conclusão dos resultados.

D5 avaliou como as contribuições de uma experiência de ensino, com foco na Teoria dos Campos Conceituais 
trouxeram para os estudantes do 30 ano do Ensino Fundamental no desenvolvimento de habilidades e competências na resolução de situações-problemas que envolvem o campo aditivo. A escola pertence à rede privada, localizada na cidade de Porto Alegre. A pesquisa é de cunho qualitativo, apresentando como abordagem o estudo de caso e a observação participante. (Silva, 2014) apresentou, como resultados, que trabalhar com situações-problema de composição e transformação pode contribuir no ensino e aprendizagem, propiciando o desenvolvimento de habilidades, competências, teoremas-em-ação e conceitos-em-ação.

D6 objetivou investigar como as tecnologias móveis (tablets e smartphones) favorecem a aprendizagem de conteúdos matemáticos do campo aditivo e multiplicativo no Ensino Fundamental. Os sujeitos foram estudantes do curso de Pedagogia e da Licenciatura em Matemática da Universidade Federal de Alagoas (UFAL). Os dados foram analisados com base nas pesquisas de Carvalho, Vergnaud, Borba e Bairral . Esta pesquisa é de cunho qualitativo na modalidade estudo de caso. Os resultados da pesquisa de (Freitas, 2017) indicou o interesse dos alunos nas possibilidades e limitações da utilização das tecnologias móveis como recursos voltados ao ensino e a aprendizagem. De igual modo, a compreensão dos campos conceituais mobiliados através da participação nas discussões e no envolvimento das atividades propostas.

T1 apresentou como objetivo identificar possibilidades didático-pedagógicas de um trabalho sistemático envolvendo o cálculo mental, de maneira dialógica. Os sujeitos foram três alunos surdos fluentes do $6^{\circ}$ ano do Ensino Fundamental. A pesquisa apresentou como embasamento teórico a Teoria dos Campos Conceituais e pesquisas que abordam a especificidade da Língua Brasileira de Sinais (LIBRAS) e do Transtorno de Déficit de Atenção e Hiperatividade (TDAH). A metodologia adotada foi a Engenharia Didática, com aplicação de uma sequência didática dividida em dois blocos, quais sejam: SND e o Aditivo. Como resultados, (Zanquetta, 2015) constatou que cada sujeito, ao se deparar com uma nova situação adaptou, a seu modo, seus conhecimentos à nova situação apresentada. A dinâmica de cálculo mental beneficiou a troca de ideias, bem como o desenvolvimento da autonomia dos estudantes. Identificou que não houve um consenso acerca dos sinais em LIBRAS para os termos matemáticos entre os profissionais inseridos no contexto educacional. No decorrer da sequência didática, não foi suficiente o uso do cálculo mental, necessitando utilizar recursos didáticos.

T2 apresentou como objetivo investigar o campo de competência em que estudantes do $6^{\circ}$ ano do Ensino Fundamental precisam ter para solucionar problemas aritméticos com os números decimais, tanto no campo aditivo quanto no multiplicativo. Os sujeitos foram alunos do $6^{\circ}$ ano do Ensino Fundamental de uma escola pública de Belém do Pará. Como metodologia, foi escolhida a pesquisa mista. Os resultados de (Jucá, 2014) mostraram que os alunos que tinham habilidades com as operações dos números naturais aprenderam de maneira significativa as operações com os números decimais, obtendo avanço nas habilidades em resolver situações-problema.

T3 objetivou analisar a ampliação de competências profissionais, bem como a resignificação do conhecimento de docentes que ensinam Matemática nos Anos Iniciais do Ensino Fundamental acerca da ideia de relações contextuais. Os sujeitos foram quatro professoras que ensinam Matemática nos Anos Iniciais. A base teórica apresentada foi sobre a formação de professores e os processos de ensino e aprendizagem das relações aditivas e multiplicativas, apresentando como principais teóricos Ball, Thames e Phelps, Vergnaud e Nunes, Bryant, Evans, Gottardis,Terlektsi, Serrazina e Zeihner, dentre outros. A metodologia utilizada foi de caráter qualitativo, apoiada nos pressupostos do Design Experiment, de caráter intervencionista. Os resultados de (Lopes, 2017) apontam que a ampliação de competências e o conhecimento profissional dos sujeitos ocorreram através da destinação de espaços interativos, estudos, reflexão da práxis em um contexto de formação continuada. Os estudos no contexto da comunidade de prática propiciaram o compartilhamento de análises de tarefas profissionais, acerca do pensamento matemático de estudantes sobre as relações contextuais no campo aditivo e multiplicativo.

A partir da apresentação das pesquisas encontradas na RSL, a seguir, discute-se os resultados. 


\section{Resultados e Discussão}

As dissertações e teses analisadas indicam $100 \%$ do uso da Teoria dos Campos Conceituais (TCC) como uma das bases teóricas utilizadas. A TCC possibilita o estudo e a compreensão das aprendizagens dos estudantes, assim como pode auxiliar o professor a entender suas limitações e dificuldades (Magina et al, 2010). Portanto, é uma Teoria que pode fornecer e subsidiar pesquisas que exploram o Campo Aditivo.

As pesquisas D1, D4, D6, T2 e T3 abordaram tanto o Campo Aditivo como o Multiplicativo, mostrando, assim como (Castro, 2016), que há interseções entre estes dois campos conceituais. As pesquisas D2, D3, D5 e T1 focaram apenas no campo aditivo. Este último campo foi uma das palavras-chaves inseridas no refinamento das pesquisas, o que já era esperado.

É importante destacar que as pesquisas D3 e D4 focaram em alunos do $5^{\circ}$ ano, enquanto que D5 em alunos do $3^{\circ}$ ano do Ensino Fundamental. D3 apresentou em seus resultados que os estudantes apresentavam dificuldades no algoritmo. Nos resultados da pesquisa $\mathrm{D} 4$, os estudantes, que também são do 5o ano, não apresentaram dificuldades no algoritmo, o que mostra a diferença no nível de aprendizagem das crianças no mesmo ano escolar. D5 apresentou os resultados dos estudantes do $3^{\circ}$ ano que trabalharam as situações-problema auxiliando no processo de aprendizagem. Conclui-se a necessidade em se trabalhar esses conceitos de maneira contínua nos anos iniciais.

T1 e T2 voltaram suas pesquisas para o $6^{\circ}$ e $7^{\circ}$ anos do Ensino Fundamental. Desta forma, é visto que estudantes dos anos mais avançados ainda apresentam dificuldades na aprendizagem de situações que envolvem adição e subtração.

Já D6 e T3 tiveram como sujeitos estudantes de graduação e professores, respectivamente. As duas pesquisas focaram na formação de professores. A diferença é que em uma os sujeitos estavam na formação inicial e na outra, formação continuada. Dessa forma, destaca-se a importância em apresentar pesquisas com professores e futuros professores sobre as temáticas supracitadas.

Tais dificuldades podem ser constatadas a partir de avaliações de larga escala, como o Sistema de Avaliação da Educação Básica (SAEB). Resultados recentes indicam que mais da metade dos alunos $(51,10 \%)$ concluíram o $5^{\circ}$ ano sem aprender a resolver situações problemas envolvendo adição e subtração (Brasil, 2019).

O SAEB apresenta os quadros com os níveis e descrições das habilidades/descritores desenvolvidas pelos estudantes que estão relacionados com os conceitos de adição e de subtração, nos quais os alunos que estão concluindo o último ano dos anos iniciais do Ensino Fundamental precisam ser capazes de apresentar habilidades/descritores, quais sejam: "Resolver problemas do cotidiano envolvendo adição de pequenas quantias de dinheiro; Resolver problemas envolvendo a análise do algoritmo da adição de dois números naturais"; (Brasil, 2019, p. 56-57).

Quanto à palavra Recurso Educacional Digital, não foram encontradas pesquisas que abordassem essa nomenclatura. Porém, pesquisas como D1 adotou o software educacional "Frame" e D6 adotou as tecnologias móveis (celulares e smartphones). Tais tecnologias apresentou avanços conceituais dos estudantes no campo aditivo, e na prática pedagógica. Apesar do termo RED não ter aparecido nas pesquisas, ele é apresentado através de imagens, áudios, vídeos, textos, animações e/ou hiperlinks (Hitzschky, 2019). Constata-se a não houveram teses e dissertações que adotaram o termo Recurso Educacional Digital nas suas pesquisas, mesmo que essa nomenclatura seja utilizada ultimamente.

É necessário afirmar que as tecnologias digitais, junto a uma metodologia adequada, podem favorecer o processo de ensino e aprendizagem, auxiliando em novas propostas colaborativas na Educação (Vieira, Lacerda, \& Moura-Junior, 2017).

Nesta perspectiva, é possível identificar que as TDIC e as mídias digitais têm causado forte impacto em todos os segmentos da sociedade atual, da nossa vida e, principalmente, no conhecimento científico, bem como nos avanços da ciência (Almeida, \& Valente, 2012).

Por fim, novamente, D1 e D6 foram as únicas pesquisas que utilizaram o Campo Aditivo e o RED em suas abordagens. Isto significa que há a necessidade no desenvolvimento de pesquisas voltadas à utilização do RED na 
aprendizagem do campo aditivo.

\section{Considerações Finais}

A pesquisa apresentou uma Revisão Sistemática de Literatura de teses e dissertações dos últimos 10 anos em que foram apresentadas como campo de pesquisa o uso de Recursos Educacionais Digitais e o ensino do Campo Aditivo. Através da busca pelo repositório de teses e dissertações da CAPES, utilizando os filtros supracitados, foram encontradas 27 pesquisas. Por não estarem inseridas no campo de pesquisa voltados para o Campo Aditivo e Recursos Educacionais Digitais, apenas 9 pesquisas foram catalogadas.

Diante da RSL realizada, na busca por responder o objetivo deste artigo, concluímos que há a necessidade de futuras pesquisas que abordem o uso do RED no ensino da adição e subtração.

Através das justificativas apresentadas nos resultados, em que mais da metade dos estudantes concluem o $5^{\circ}$ ano sem saber resolver situações aditivas, faz-se necessário pensar em metodologias que apresentem aos professores esses dados. Com isso, destaca-se a importância em apresentar o RED como uma ferramenta que apoiará o docente, bem como o estudante no que tange o ensino e aprendizagem de conceitos matemáticos.

Espera-se que este artigo possa influenciar futuras pesquisas que apresentem a utilização de RED por professores no ensino do campo aditivo. Como pretensão para trabalhos futuros, propõe-se uma investigação científica e tecnológica, nesse sentido, utilizando de recursos educacionais digitais e o ensino do campo aditivo aplicados no ambiente escolar.

\section{Referências}

Afonso, M. C. L., Eirão, T. G., Melo, J. H. M., Assunção, J. S., \& Leite, S. V. (2011). Banco Internacional de Objetos Educacionais (BIOE): tratamento da informação em um repositório educacional digital. Perspectivas em Ciência da Informação, 16(3), 148-158.

Almeida, M. E. E. (2016). Currículo e narrativas digitais em tempos de ubiquidade: criação e integração entre contextos de aprendizagem. Revista de Educação Pública, 25(59/2), 526-546. https://doi.org/10.29286/rep.v25i59/2.3833.

Amiel, T., \& Soares, T. (2015). Chagas. O contexto da abertura: recursos educacionais abertos, cibercultura e tensões. Em Aberto, Brasília, 28(94), 109-122. http://rbepold.inep.gov.br/index.php/emaberto/article/view/1673/1644

Anjos, D. R. K. (2014). Uma análise praxeológica das operações de adição e subtração de números naturais em uma coleção de livros didáticos dos anos iniciais do ensino fundamental, 142 f. Dissertação (Mestrado) - Curso de Programa de Pós-Graduação em Educação Matemática, Universidade Federal do Mato Grosso do Sul, Campo Grande.

Braga, J., \& Menezes, L. (2014). Introdução aos Objetos de Aprendizagem. https://pesquisa.ufabc.edu.br/intera/wp-content/uploads/2015/12/objetos-deaprendizagem-v1.pdf.

Brasil. M. E. (2017). Base Nacional Comum Curricular: Educação é a base. http://basenacionalcomum.mec.gov.br/images/ BNCC_publicacao.pdf .

Brasil. M. E. (2019). Relatório SAEB 2017. Brasília, DF: Instituto Nacional de Estudos e Pesquisas Educacionais Anísio Teixeira. Brasil, 2019. http://portal.inep.gov.br/informacao-da-publicacao/-/asset_publisher/6JYIsGMAMkW1/document/id/6730262 .

Caiado, R, Rangel, L., Quelhas, O. L. G., \& Nascimento, D. (2016). Metodologia de Revisão Sistemática de Literatura com aplicação do método de apoio multicritério à decisão smarter. https://www.inovarse.org/sites/default/files/T16_002.pdf.

Castro, J. B. (2012). A utilização de objetos de aprendizagem para a construção e compreensão de gráficos estatísticos. Dissertação (Mestrado em Educação) - Universidade Federal do Ceará, Fortaleza. http://www.repositorio.ufc.br/handle/riufc/7341 .

Castro, J. B. (2016). Construção do conceito de covariação por estudantes do ensino fundamental em ambientes de múltiplas representações com suporte das tecnologias digitais. 2016. 275f. - Tese (Doutorado) - Universidade Federal do Ceará, Programa de Pós-graduação em Educação Brasileira, Fortaleza (CE). http://www.repositorio.ufc.br/handle/riufc/15908 .

Castro Filho, J. A., Freire, R. S., Fernandes, A. C., \& Leite, M. A. (2008). Quando objetos digitais são efetivamente para aprendizagem: o caso da matemática. Brazilian Symposium on Computers in Education (Simpósio Brasileiro de Informática na Educação - SBIE), 583-592. https://www.brie.org/pub/index.php/sbie/article/view/747/733.

Fiore, C. A. (2013). Os pensamentos narrativos e lógico científico na resolução de problemas nos campos conceituais aditivo e multiplicativo no ano final do ensino fundamental I. Dissertação (MESTRADO) - Universidade Bandeirante Anhanguera.

Freitas, R. O. (2017) Tecnologias móveis na formação de professores que ensinam matemática. Dissertação (Mestrado em Educação) - Centro de Educação, Programa de Pós Graduação em Educação, Universidade Federal de Alagoas, Maceió. 
Galvão, M. C. B., \& Ricarte, I. L. M. (2019). Revisão Sistemática da Literatura: conceituação, produção e publicação. Logeion: Filosofia da Informação, 6(1), 57-73. http://revista.ibict.br/fiinf/article/view/4835. 10.21728/logeion.2019v6n1.p57-73.

Hitzschky, R. A. (2019). Desenvolvimento de um Recurso Educacional Digital (RED) de Língua portuguesa fundamentado na base nacional comum Curricular. Dissertação (Mestrado) - Universidade Federal do Ceará, Programa de Pós-graduação em Educação, Fortaleza(CE).

Hodges, C. B., Moore, S., Lockee, B. B., Trust, T., \& Bond, M. A. (2020). The difference between emergency remote teaching and online learning. Educause Review. https://er.educause.edu/articles/2020/3/the-difference-between-emergency-remote-teaching-and-online-learning.

Jucá, R. S. (2014). Um estudo das competências e habilidades na resolução de problemas aritméticos aditivos e multiplicativos com os números decimais. Tese (Doutorado em Educação, em Ciências e Matemática) -- Universidade Federal do Pará, Instituto de Educação Matemática e Cientifica, Programa de Pósgraduação da Rede Amazônica em Educação, em Ciências e Matemática, Belém.

Lira, A. S., Leitão, D. A., \& Castro, J. B. (2019). Como o Processo de Produção de Mídias pode Contribuir para a Formação Docente? Renote. Revista Novas Tecnologias Na Educação, 17, 425-434. https://seer.ufrgs.br/renote/article/viewFile/95850/53869 .

Lopes, S. C. (2017). Comunidade de prática para o desenvolvimento de competências profissionais voltadas para a resolução de problemas matemáticos de relações contextuais. Tese (Programa de Pós-graduação em Educação Matemática) - Coordenadoria de Pós-graduação - Universidade Anhanguera de São Paulo.

Magina, S. P., Santana, E. R. S., Cazorla, I. M., \& Campos, T. M. M. (2010). As Estratégias de Resolução de Problemas das Estruturas Aditivas nas Quatro Primeiras Séries do Ensino Fundamental. Zetetiké, Unicamp, 18(34), 15-50.

Pereira, J. F. F. (2013). Resolução de problemas do campo aditivo por alunos de quinto ano de uma escola pública da cidade de São Paulo. Dissertação (mestrado) - Programa de Pós-Graduação em Ensino de Ciências e Matemática, Universidade Cruzeiro do Sul.

Ravindran, V., \& Shankar, S. (2015). Systematic reviews and meta-analysis demystified. Indian J.Rheumatol. 10, 89-94. 2015.

Rojo, R., \& Moura, E. (2012). Multiletramentos na Escola. Parábola.

Santos, D. B. (2015). Ensino de Matemática crítico e reflexivo: A teoria das situações didáticas como estratégia para a aprendizagem das operações básicas da aritmética na rede municipal de ensino. Dissertação (Mestrado) - Curso de Mestrado Profissional Gestão e Tecnologia Aplicada À Educação - Gestec, Universidade do Estado da Bahia, Salvador.

Silva, G. B. (2014). Teoria dos Campos Conceituais, Habilidades e Competências: uma experiência de ensino. Dissertação (Mestrado) - Curso de Programa de Pós-Graduação em Educação, Centro Universitário La Salle, Canoas.

Souza, E. (2015). Estruturas multiplicativas: concepção de professor do ensino fundamental. Dissertação (Mestrado) - Universidade Estadual de Santa Cruz. Programa de Pós- graduação em Educação Matemática.

Vergnaud, G. (2009). A criança, a matemática e a realidade: problemas do ensino da matemática na escolar elementar. Ed. da UFPR.

Vergnaud, G. (1996). A Teoria dos Campos conceituais. In. Brun, J. Didáctica das matemáticas. Tradução por Maria José Figueiredo. Lisboa: Instituto Piaget, $155-191$.

Vieira, F., Lacerda, M., \& Moura-Junior, A. (2017). Reflexões sobre avaliação de games educacionais. Anais do Seminário de Jogos Eletrônicos, Educação e Comunicação, Salvador, 2(2).

Zanquetta, M. E. M. T. (2015). Uma investigação com alunos surdos do Ensino Fundamental: o cálculo mental em questão. Tese (Doutorado em Programa de Pós-Graduação em Educação para ciência e a matemática). Universidade Estadual de Maringá. 